\title{
Flank Pain Could be a COVID-19 Messenger !
}

\author{
emrullah durmus ${ }^{1}$, fesih ok $^{2}$, omer erdogan ${ }^{1}$, and semih saglik ${ }^{1}$ \\ ${ }^{1}$ Affiliation not available \\ ${ }^{2}$ Siirt Training and Research Hospital
}

August 23, 2020

\begin{abstract}
Aim: We aimed to present the patients who had COVID-19 infiltrations incidentally detected in the lung basal sections in patients with abdomen $\mathrm{CT}$ due to flank pain in the urology outpatient clinic during the pandemic process. Methods: A total of 276 patients admitted to the Siirt Training and Research Hospital Urology outpatient clinic between March 15, 2020, and August 09, 2020, with a complaint of flank pain and underwent non-contrast abdomen CT were analyzed from this data retrospectively. A total of 10 patients with Covid-19 compatible findings in CT were determined as the study group. A control group was formed from 10 patients with only urological pathologies (kidney stones, ureteral stones, hydronephrosis ... etc) without Covid-19 compatible appearance on CT. Results: Ten (3.6\%) patients with COVID-19 disease pneumonic infiltrations were detected in the lung basal regions entering the abdomen CT cross-section. The visual analog scale (VAS) score was higher in the control group, who had urological pathology and did not have Covid-19 findings in the lung bases in abdominal CT, and it was statistically significant. During the admission to the urology outpatient clinic, there were no signs of COVID-19 disease such as fever, cough, and shortness of breath. Conclusion: If the severity of pain is not very high in patients who apply to urology outpatient clinics with flank pain during the pandemic period, and if no urological pathology is observed in the Abdomen CT, Covid-19 should be kept in mind in the differential diagnosis.
\end{abstract}

Title: Flank Pain Could be a COVID-19 Messenger !

Aim: We aimed to present the patients who had COVID-19 infiltrations incidentally detected in the lung basal sections in patients with abdomen CT due to flank pain in the urology outpatient clinic during the pandemic process.

Methods : A total of 276 patients admitted to the Siirt Training and Research Hospital Urology outpatient clinic between March 15, 2020, and August 09, 2020, with a complaint of flank pain and underwent noncontrast abdomen CT were analyzed from this data retrospectively. A total of 10 patients with Covid-19 compatible findings in CT were determined as the study group. A control group was formed from 10 patients with only urological pathologies (kidney stones, ureteral stones, hydronephrosis ... etc) without Covid-19 compatible appearance on CT.

Results: Ten (3.6\%) patients with COVID-19 disease pneumonic infiltrations were detected in the lung basal regions entering the abdomen CT cross-section. The visual analog scale (VAS) score was higher in the control group, who had urological pathology and did not have Covid-19 findings in the lung bases in abdominal CT, and it was statistically significant. During the admission to the urology outpatient clinic, there were no signs of COVID-19 disease such as fever, cough, and shortness of breath.

Conclusion: If the severity of pain is not very high in patients who apply to urology outpatient clinics with flank pain during the pandemic period, and if no urological pathology is observed in the Abdomen CT, Covid-19 should be kept in mind in the differential diagnosis.

Key words: COVID-19, urology, flank pain, Abdomen CT 


\section{What's known}

* Covid-19 disease may present with an unusual symptom.

* Early detection of computed tomography findings in Covid-19 disease is important to prevent the spread of the disease in the society.

\section{What's new}

* Non-severe flank pain may be a sign of Covid-19

* This is the first study in the literature that examines the abdominal CT images of patients presenting with flank pain in terms of COVID-19 disease.

\section{INTRODUCTION}

In December 2019, Wuhan, the capital of China's Hubei region, began to experience cases of pneumonia that did not respond to standard treatments. A new coronavirus called SARS-CoV-2 was detected as the cause of the disease ${ }^{1}$. The SARS-CoV-2 is a $\beta$-coronavirus, which is enveloped non-segmented positive-sense RNA virus $^{2}$. The disease spread rapidly worldwide. On March 11, 2020, the world health organization (WHO) declared that this was a pandemic ${ }^{3}$. In Turkey until the date August 09, 2020, the number of confirmed cases was 240804 , and the number of recovered cases were 223759 . The number of patients who died due to the virus was 5844. The COVID-19 disease is highly contagious and can rapidly progress to Acute respiratory distress syndrome (ARDS), leading to death ${ }^{4,5}$. Older men with comorbidity are more likely to have respiratory failure, and some patients have made rapid progress to multi-organ dysfunction ${ }^{6}$. The Centers for Disease Control and Prevention (CDC) recommends that after two negative respiratory tests separated by [?] 24 hours, patients can be dismissed from having transmissibility infection risk for COVID19. In laboratory examination results, most patients had normal or decreased white blood cell counts, and lymphocytopenia ${ }^{7,8}$.

CT findings are critical in the diagnosis of COVID-19 disease. The imaging findings of COVID-19 disease are not specific and variable. However, most common findings are round pulmonary parenchymal ground-glass opacities without lung cavitation, separate pulmonary nodules or pleural effusion ${ }^{9}$. Other less common imaging features include linear densities, pavement pattern, bronchial wall thickening and reverse halo sign 10 .

In this study, we aimed to present patients who admitted to our urology outpatient clinic with a complaint of flank pain during the pandemic process and incidentally have ground-glass densities compatible with COVID-19 in basal lung sections on abdomen CT images.

\section{PATIENTS AND METHODS}

The Institutional Ethical Board approved this study of Siirt University (Approval Number 2020/08.01). In our routine practice, patients with a history of urolithiasis in the family or themselves and who have the findings to support the stone in laboratory examinations, generally, non-contrast Abdomen CT is performed. Data of patients with flank pain are routinely recorded in the Microsoft Excel ${ }^{\circledR}$ program in our hospital's urology clinic. The severity of the pain felt by patients presenting with flank pain is scored and recorded from one to ten using the visual analog scale (VAS) pain scoring system (Figure 1). A total of 276 patients admitted to the Siirt Training and Research Hospital Urology outpatient clinic between March 15, 2020, and August 09, 2020, with a complaint of flank pain and underwent non-contrast abdomen CT were analyzed from this data retrospectively, accompanied by a radiologist with 5 years of post-fellowship experience. Abdomen CT images of the patients were analyzed using the Siso-Pacs ${ }^{\circledR}$ image archiving and communication system. Abdomen CT images were reexamined in the lung parenchyma window and patients ground-glass density compatible with Covid-19 findings in the lung bases entering the section were included in the study. In addition, A total of 10 patients with Covid-19 compatible findings in CT were determined as the study group. A control group was formed from 10 patients with only urological pathologies (kidney 
stones, ureteral stones, hydronephrosis ... etc) without Covid-19 compatible appearance on CT. The two groups were compared statistically according to the selected parameters.

Patients with respiratory symptoms at admission, and only those with ultrasound (US) imaging were excluded. Also, the negative results of the polymerase chain reaction (PCR) test were excluded from the study.

\section{Statistical Analysis}

SPSS Statistics software version 25.0 (IBM, Armonk, NY) was used for all statistical analyses. Shapiro Wilk test was used to test the normal distribution of numerical variables. Continuous variables were specified as appropriate means and standard deviations or medians and interquartile ranges. The $\chi 2$ and Fisher's exact tests were applied to categorical variables. The Student T-test and Mann-Whitney U tests were used for continuous variables. $\mathrm{P}$ value of less than 0.05 was defined as statistical significance.

\section{RESULTS}

Ten $(3.6 \%)$ patients with COVID-19 disease pneumonic infiltrations were detected in the lung basal regions entering the abdomen CT cross-section. Three patients had unilateral (Figure 2) and seven patients had bilateral infiltration (Figure 3). The male to female ratio was $6 / 4$. The mean age was $52.4 \pm 21.6$ years. Four patients had right flank pain, Four patients had left flank pain and two patient had bilateral flank pain. In one patient, two 5-mm-sized stones in the right kidney and a 5-mm-sized stone in the middle part of the right ureter were detected. One patient had a bilateral grade one hydronephrosis. In the other eight patients, no urological pathology was observed in Abdomen CT. A 16-year-old patient with complaints of left flank pain reflected in the left testicle had no stone in Abdomen CT, and left epididymo orchitis was detected in Scrotal Doppler Ultrasonography. During the admission to the urology outpatient clinic, there were no signs of COVID-19 disease such as fever, cough, and shortness of breath. PCR test was positive in all patients. The median time the patients applied to the covid-19 outpatient clinic with another symptom after leaving the urology outpatient clinic was 2.4 (1-5) days. The median VAS score of patients with Covid-19 findings on abdominal CT was 4 points. The median VAS score of patients with urological pathology without Covid-19 findings was found 8 points. The VAS score was higher in the control group, who had urological pathology and did not have Covid-19 findings in the lung bases in abdominal CT, and it was statistically significant $(\mathrm{p}<0.001)$ (Table 1).

\section{DISCUSSION}

The COVID-19 disease is spreading rapidly worldwide, and no vaccine or very effective drug has been found yet. However, the transmission rate of the disease can be reduced by precautions to be taken. Therefore, it is essential to detect the patients early to prevent the spread of the disease ${ }^{11,12}$. In addition to supportive treatments, medical treatments (antivirals, antibiotics, corticosteroids, hydroxychloroquine etc.) are applied. Unfortunately, a standard treatment protocol applied worldwide has unfortunately not yet been developed. Many treatment options have been tried in China and other countries and their effects on the virus have been published. In another study had identified 4 small molecular drugs (prulifloxacin, nelfinavir, bictegravir, tegobuvir) with high binding capacity with SARS-CoV-2 main protease ${ }^{13}$. It has been shown that agents such as remdesivir, chloroquine and baricitinib can be used in the treatment of covid-19 ${ }^{14,15}$. Although the most common symptom of COVID-19 disease is fever, cough, and shortness of breath are also considered among other common symptoms ${ }^{16,17}$. Uncommon symptoms such as abdominal pain, diarrhea, and olfactory disorders have also been reported in the literature as a first-line symptom ${ }^{18-20}$. However, flank pain is not as joint as a first-line symptom. According to the literature review, there are no studies examining the abdominal CT images of patients presenting with flank pain in terms of COVID-19 disease.

The sensitivity of ultrasonography in detecting urinary stones varies from $3 \%$ to $98 \%$ in various studies ${ }^{21,22}$. Ultrasonography can be challenging in obese patients and generally offers limited or poor visualization of the mid ureter. In addition, Ultrasonography has a limited ability to depict renal or ureteral calculi which are smaller than $5 \mathrm{~mm}$. However, detection of calculi smaller than $5 \mathrm{~mm}$ is of questionable clinical significance, as such patients are unlikely to require urological intervention ${ }^{23}$. Many studies have shown that non-contrast 
abdominal CT is unlikely to miss stones that require intervention compared to Ultrasound ${ }^{24,25}$. Recent data suggest that less than $7 \%$ of patients diagnosed with kidney stones are using Ultrasonography and the use of CT continues to increase ${ }^{26}$. The American College of Radiology Appropriateness Criteria states that a low-dose non-enhanced $\mathrm{CT}$ of the abdomen and pelvis is the imaging examination of choice for evaluating patients with suspected urolithiasis (sensitivity $97 \%$ and specificity $95 \%$ ) $^{27}$. Non-contrast Abdomen CT can sometimes be the first imaging method used in patients with severe renal $\operatorname{colic}^{28}$.

The prevalence of the stone disease is very high in our region. For this reason in our clinic patients with a history of urolithiasis in the family or themselves and who have the findings to support the stone in laboratory examinations, generally, to detect suspected kidney and ureteral stones that cannot be detected on the Ultrasonography, non-contrast Abdomen CT is performed.

In one of the current publications on COVID-19 and CT imaging, the sensitivity of Thorax CT (98\%) in the early diagnosis of COVID-19 was reported to be higher than that of the RT-PCR test $(71 \%)^{29}$. This information increases the importance of Thorax CT in the diagnosis of the disease. During pandemic period in our country regardless of the branch, all physicians began to take care of COVID-19 patients. During this period, our experience with the Thorax CT imaging method, which we usually do not use as a urology specialist, has increased. We had the opportunity to see and evaluate many Thorax CTs. In this way, we learned to assess the basal lung parts of the abdominal CTs, which we pulled for urological reasons, in the lung parenchyma window. For COVID-19 disease the most common findings of Thorax CTs are round pulmonary parenchymal ground-glass opacities. Abdominal and back pain has long been described as a symptom in pneumonia in both adults and children secondary to pleural irritation ${ }^{30,31}$. When the patients who applied to the urology outpatient clinic during the pandemic period were screened backward, we found that ten patients $(3.6 \%)$ presented with flank pain and that there were COVID-19 compatible pneumonic infiltrations in the lung bases in the abdominal CT. Irritation-related flank pain caused by inflammation in the lung basal may be the first symptom of COVID-19 disease in some patients. The first diagnosis that comes to mind in a patient admitted to the urology outpatient clinic with flank pain is stone disease. However, In this period when the COVID-19 pandemic continues, It should be kept in mind that if the pain score according to the visual analog scale pain scoring system is not very high in patients presenting with flank pain and there is no urological pathology in Abdomen CT, this pain may be a Covid-19 symptom. At the same time, radiologists should not overlook Covid-19 when evaluating images in Abdomen CTs taken in normal clinics other than the pandemic outpatient clinic. Unfortunately, there is no more effective option to struggle the Covid-19 pandemic than to reduce contagion.

The limitation of our study is that it was performed retrospectively in a single center and with a limited number of patients. Multicenter and comprehensive studies are needed to strongly advocate this outcome.

\section{CONCLUSION}

If the severity of pain is not very high in patients who apply to urology outpatient clinics with flank pain during the pandemic period, and if no urological pathology is observed in the Abdomen CT, Covid-19 should be kept in mind in the differential diagnosis. Before the radiologist report, Clinicians should be knowledgeable enough to evaluate the basal parts of the lung entering the cross-sectional area in terms of COVID-19 disease while examining the Abdomen CT. Thus, Clinicans can protect themselves and other healthcare workers from transmission during the examination. In this way, patients can be caught early, before severe COVID-19 symptoms begin.

\section{ETHICAL APPROVAL}

Informed consent was obtained from all individual participants included in the study. All procedures performed in studies were in accordance with the ethical standards of the institutional and/or national research committee and with the 1964 Helsinki declaration and its later amendments or comparable ethical standards.

\section{CONFLICTS OF INTEREST}

All authors have no conflict of interest to declare 


\section{REFERENCES}

1. The Lancet. Emerging understandings of 2019-nCoV. Lancet . 2020;395(10221):311. doi:10.1016/S0140$6736(20) 30186-0$

2. Zhu N, Zhang D, Wang W, Li X, Yang B, Song J, et al. A novel coronavirus from patients with pneumonia in China, 2019. N Engl J Med. 2020;382(8):727-733. doi: 10.1056/NEJMoa2001017.

3. World Health Organisation (WHO) Coronavirusdisease (COVID-19) Outbreakwebpage. Available at: Https://Www. Who.Int/Emergencies/Diseases/Novel-Coronavirus-2019 .

4. Li Q, Guan X, Wu P, et al. Early transmission dynamics in Wuhan, China, of novel coronavirus-infected pneumonia. N Engl J Med . 2020;382(13):1199-1207. doi:10.1056/NEJMoa2001316

5. Wang D, Hu B, Hu C, et al. Clinical Characteristics of 138 Hospitalized Patients with 2019 Novel Coronavirus-Infected Pneumonia in Wuhan, China. JAMA - J Am Med Assoc . 2020;323(11):1061-1069. doi:10.1001/jama.2020.1585

6. Huang C, Wang Y, Li X, et al. Clinical features of patients infected with 2019 novel coronavirus in Wuhan, China. Lancet . 2020;395(10223):497-506. doi:10.1016/S0140-6736(20)30183-5

7. Guan WJ, Ni ZY, Hu Y, Liang WH, Ou CQ, He JX, et al. Clinical characteristics of coronavirus disease 2019 in China. N Engl J Med. 2020. 10.1056/NEJMoa2002032

8. Kui L, Fang YY, Deng Y, Liu W, Wang MF, Ma JP, et al. Clinical characteristics of novel coronavirus cases in tertiary hospitals in Hubei Province. Chin Med J. 2020. 10.1097/CM9.0000000000000744

9. Chung M, Bernheim A, Mei X, et al. CT imaging features of 2019 novel coronavirus (2019-NCoV). Radiology . 2020;295(1):202-207. doi:10.1148/radiol.2020200230

10. Bernheim A, Mei X, Huang M, et al. Chest CT findings in coronavirus disease 2019 (COVID-19): Relationship to duration of infection.Radiology . 2020;295(3):685-691. doi:10.1148/radiol.2020200463

11. Chan JFW, Yuan S, Kok KH, et al. A familial cluster of pneumonia associated with the 2019 novel coronavirus indicating person-to-person transmission: a study of a family cluster. Lancet . 2020;395(10223):514-523. doi:10.1016/S0140-6736(20)30154-9

12. Phan LT, Nguyen T V., Luong QC, et al. Importation and human-to-human transmission of a novel coronavirus in Vietnam. N Engl J Med . 2020;382(9):872-874. doi:10.1056/NEJMc2001272

13. Li Y, Zhang J, Wang N, et al. Therapeutic drugs targeting 2019-ncov main protease by highthroughput screening. bioRxiv. 2020. doi: 2020.01.28.922922.

14. Wang M, Cao R, Zhang L, et al. Remdesivir and chloroquine effectively inhibit the recently emerged novel coronavirus (2019-nCoV) in vitro. Cell Res. 2020;30:269-271.

15. Richardson P, Griffin I, Tucker C, et al. Baricitinib as potential treatment for 2019-nCoV acute respiratory disease. Lancet. 2020;395:e30-e31.

16. Wang W, Tang J, Wei F. Updated understanding of the outbreak of 2019 novel coronavirus (2019-nCoV) in Wuhan, China. J Med Virol . 2020;92(4):441-447. doi:10.1002/jmv.25689

17. Xie X, Zhong Z, Zhao W, Zheng C, Wang F, Liu J. Chest CT for Typical 2019-nCoV Pneumonia: Relationship to Negative RT-PCR Testing.Radiology . Published online February 12, 2020:200343. doi:10.1148/radiol.2020200343

18. Wong SH, Lui RNS, Sung JJY. Covid-19 and the digestive system.J Gastroenterol Hepatol . 2020;35(5):744-748. doi:10.1111/jgh.15047

19. Song Y, Liu P, Shi XL, et al. SARS-CoV-2 induced diarrhoea as onset symptom in patient with COVID19. Gut . 2020;69(6). doi:10.1136/gutjnl-2020-320891 
20. Lechien JR, Chiesa-Estomba CM, De Siati DR, et al. Olfactory and gustatory dysfunctions as a clinical presentation of mild-to-moderate forms of the coronavirus disease (COVID-19): a multicenter European study. Eur Arch Oto-Rhino-Laryngology . Published online 2020. doi:10.1007/s00405-020-05965-1

21. Hamm M, Wawroschek F, Weckerman D, et al. Unenhanced helical computed tomography in the evaluation of acute flank pain. Eur Urol. 2001;39:460-465.

22. Abdel-Gawad M, Kadasne R, Anjikar C, et al. Value of color Doppler ultrasound, KUB and urinalysis in diagnosis of renal colic due to ureteral stones. Int Braz J Urol. 2014;40:513-519.

23. Smith-Bindman R, Aubin C, Bailitz J, et al. Ultrasonography versus computed tomography for suspected nephrolithiasis. N Engl J Med 2014;371:1100e10

24. Ekici S, Sinanoglu O. Comparison of conventional radiography combined with ultrasonography versus nonenhanced helical computed tomography in evaluation of patients with renal colic. Urol Res. 2012;40:543547

25. Ripollés T, Agramunt M, Errando J, et al. Suspected ureteral colic: plain film and sonography vs unenhanced helical CT. A prospective study in 66 patients. Eur Radiol. 2004;14:129-136

26. Chang H, Dai J, Holt S, et al. National imaging trends for acute kidney stone disease: do renal ultrasounds for nephrolithiasis in the emergency department pave the way to computerized tomography? J Urol. 2018;199:e683-e684.

27. Coursey CA, Casalino DD, Remer EM, et al. ACR Appropriateness Criteria acute onset flank painesuspicion of stone disease. Ultrasound Q 2012;28:227e33.

28. Schoenfeld EM, Pekow PS, Shieh MS, et al. The diagnosis and management of patients with renal colic across a sample of US hospitals: high CT utilization despite low rates of admission and inpatient urologic intervention. PLoS One. 2017;12: e0169160.

29. Fang Y, Zhang H, Xie J, et al. Sensitivity of Chest CT for COVID-19: Comparison to RT-PCR. Radiology . Published online February 19, 2020:200432. doi:10.1148/radiol.2020200432

30. Gauss H. Abdominal pain in pneumonia. Jour D D 1946;13:73-7. https://doi.org/ 10.1007/BF03002754.

31. Grief SN, Loza JK. Guidelines for the evaluation and treatment of pneumonia. Prim

TABLE 1 Patients Characteristics and clinical features

\begin{tabular}{|c|c|c|c|c|}
\hline Charecteristics & $\begin{array}{l}\text { Covid-19 Group } \\
(\mathrm{n}=10)\end{array}$ & $\begin{array}{l}\text { Control Group } \\
(\mathrm{n}=10)\end{array}$ & Total $(n=20)$ & $P$ value \\
\hline Age, mean \pm SD & $52.4 \pm 21.6$ & $51.3 \pm 20$ & $51.8 \pm 20.3$ & 0.908 \\
\hline $\begin{array}{l}\text { Gender, n (\%) Male } \\
\text { Female }\end{array}$ & $6(60) 4(40)$ & $5(50) 5(50)$ & $11(55) 9(45)$ & 0.653 \\
\hline $\begin{array}{l}\text { Comorbidities, } \mathrm{n} \\
\text { (\%) None HT DM }\end{array}$ & $7(70) 2(20) 1(10)$ & $6(60) 1(10) 3(30)$ & $13(65) 3(15) 4(20)$ & 0.494 \\
\hline $\begin{array}{l}\text { Flank pain } \\
\text { laterality, n (\%) } \\
\text { Right Left Bilateral }\end{array}$ & $4(40) 4(40) 2(20)$ & $5(50) 4(40) 1(10)$ & $9(45) 8(40) 3(15)$ & 0.801 \\
\hline $\begin{array}{l}\text { Urinary Pathology } \\
\text { in abdomen CT,n } \\
\text { (\%) Yes No }\end{array}$ & $2(20) 8(80)$ & $10(100) 0$ & $12(60) 8(40)$ & 0.001 \\
\hline $\begin{array}{l}\text { Lung involvement } \\
\text { in abdomen CT, } \\
\text { n (\%) Unilateral } \\
\text { Bilateral }\end{array}$ & $3(30) 7(70)$ & 00 & $3(15) 7(35)$ & \\
\hline
\end{tabular}




\begin{tabular}{lllll}
\hline Charecteristics & $\begin{array}{l}\text { Covid-19 Group } \\
(\mathrm{n}=10)\end{array}$ & $\begin{array}{l}\text { Control Group } \\
(\mathrm{n}=10)\end{array}$ & Total $(\mathrm{n}=20)$ & P value \\
\hline $\begin{array}{l}\text { VAS sore, median } \\
\text { (IQR) }\end{array}$ & $4(0.5)$ & $8(2)$ & $6(4)$ & $<\mathbf{0 . 0 0 1}$ \\
\hline
\end{tabular}

HT: Hypertension, DM: Diabetes mellitus, VAS: visual analog scale, IQR: interquartile range

FIGURE 1 Visual analog scale pain scoring

FIGURE 2 Shows unilateral basal pneumatic infiltrations. 54-year-old male who admitted with right flank pain symptom. Abdominal non-contrast CT showsground-glass density compatible with COVID-19 in the right lung base (A).16-year-old male who presented with left flank pain and left testicle pain. Axial Abdomen CT demonstrates nodular ground-glass density compatible with COVID-19 in the left lung base section (B).

FIGURE 3 Demonstrate bilateral basal lung pneumatic involvements. 47-year-old male with left flank pain. A basal thoracic section of Abdominal CT. In bilateral lung basal sections, consolidation areas with ground-glass density compatible with COVID-19. Subpleural involvement on the left side (A). 68-year-old female right flank pain complaint. Axial Abdominal CT shows peribronchial thickening in the bilateral lung basal sections and consolidations in the subpleural areas as peribronchial ground-glass regions (B).

\begin{tabular}{|c|c|c|c|c|c|c|c|c|c|}
\hline \multicolumn{10}{|c|}{ VISUAL ANALOGUE SCALE } \\
\hline 1 & 2 & 3 & 4 & 5 & 6 & 7 & 8 & 9 & 10 \\
\hline $1 \quad 1$ & 1 & I & 1 & I & I & I & I & T & I \\
\hline NOPAIN & & oying & & $\begin{array}{l}\text { com } \\
\text { oder }\end{array}$ & $\begin{array}{l}\text { table } \\
\text { ) }\end{array}$ & & $\begin{array}{l}\text { orrible } \\
\text { evere) }\end{array}$ & & $\begin{array}{l}\mathrm{W} \\
\mathrm{O} \\
\mathrm{R} \\
\mathrm{S} \\
\mathrm{T}\end{array}$ \\
\hline
\end{tabular}
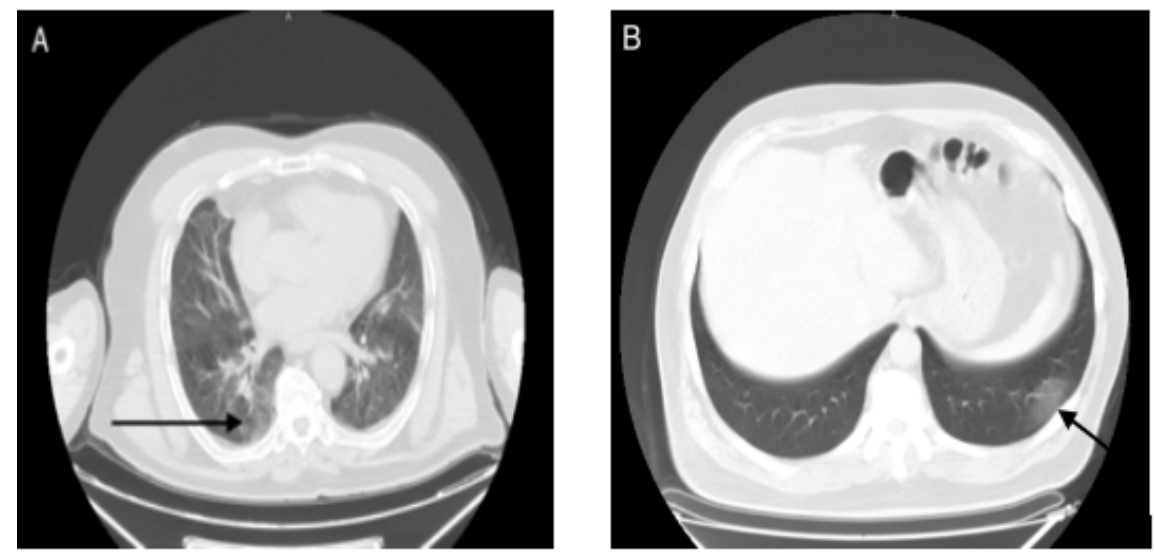

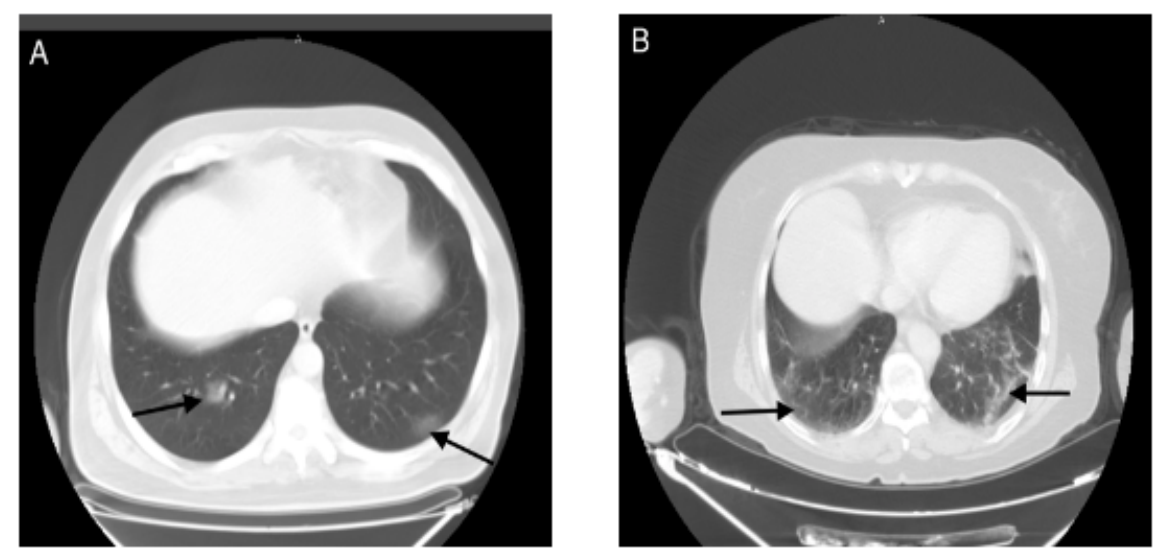